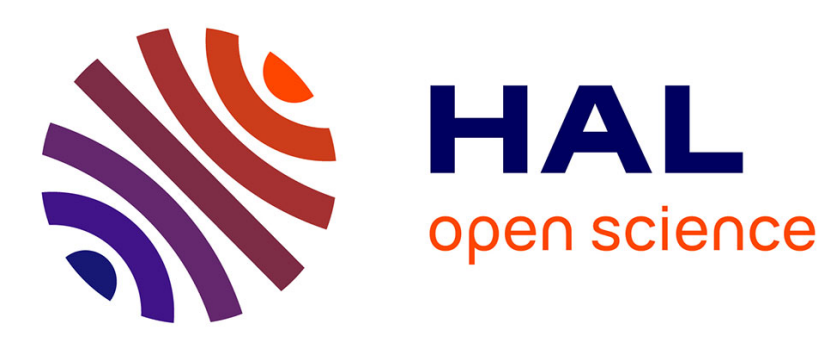

\title{
Approche de la flexibilité cognitive dans la problématique de la résolution de problème
}

Evelyne Clément

\section{To cite this version:}

Evelyne Clément. Approche de la flexibilité cognitive dans la problématique de la résolution de problème. Annee Psychologique, 2006. hal-02004920

\section{HAL Id: hal-02004920 \\ https://hal-cyu.archives-ouvertes.fr/hal-02004920}

Submitted on 2 Feb 2019

HAL is a multi-disciplinary open access archive for the deposit and dissemination of scientific research documents, whether they are published or not. The documents may come from teaching and research institutions in France or abroad, or from public or private research centers.
L'archive ouverte pluridisciplinaire HAL, est destinée au dépôt et à la diffusion de documents scientifiques de niveau recherche, publiés ou non, émanant des établissements d'enseignement et de recherche français ou étrangers, des laboratoires publics ou privés. 


\title{
Approche de la flexibilité cognitive dans la problématique de la résolution de problème
}

\author{
Évelyne Clément* \\ Laboratoire de Psychologie et Neurosciences de la Cognition, Université de Rouen
}

\section{RÉSUMÉ}

Cet article propose une relecture de la notion de flexibilité cognitive. Après être revenu sur les liens entre flexibilité, rigidité et persévération, on propose une approche de la flexibilité dans la problématique de la résolution de problème qui permet de rendre compte des différentes formes de flexibilité et des conduites de persévération. Cette approche est illustrée dans les problèmes de jarres de Luchins. On montre comment dans un même cadre conceptuel, il est possible d'interpréter les phénomènes de fixation décrits par les gestaltistes et les conduites de persévération rapportées en neuropsychologie.

Remerciements. Je tiens à remercier Jean-François Richard pour sa lecture attentive et ses remarques d'une première version du texte ainsi que Michel Piolat pour l'échange sur la perspective gestaltiste.

\section{Flexibility and problem solving: A contribution}

\begin{abstract}
This paper is a contribution to the definition of a complex behavioral process: the cognitive flexibility. By examining the literature, the different definitions lead to conclude that this notion is highly influenced by the tests used to measure a flexible behavior. We attempt to unify this notion and present an approach of the cognitive flexibility in the framework of the problem solving situations. These situations allow to identify two forms of flexibility, reactive and spontaneous flexibility, and the expression of perseverative behavior. We show how the well-known "water-jug volume-measuring problems" (Luchins, 1942) are well-suited to observe both these kind of flexibility. In those situations, spontaneous flexibility is interpreted as the capacity of adopting spontaneously different points of view on a same situation, even when reactive flexibility occurs in impasse situations and may lead either to the change of the procedure or to the elaboration of a new representation. In conclusion, we discuss how this approach permits to reinterpret in the same framework the phenomena of the fixation described by the Gestalt psychology and the perseverative behavior related in the more recent neuropsychological literature.
\end{abstract}

*Université de Rouen, Laboratoire de Psychologie et Neurosciences de la Cognition (EA 1780), 76821 Mont Saint Aigan Cedex. E-mail : evelyne.clement@univ-rouen.fr 


\section{INTRODUCTION}

La flexibilité est sans aucun doute une propriété partagée par la plupart des êtres vivants et dont la fonction est l'adaptation aux changements de l'environnement. Les défauts de flexibilité, décrits dans les premières décennies du $\mathrm{xx}^{\mathrm{e}}$ siècle comme une rigidité de la conduite, attestent de l'importance de la flexibilité dans l'adaptation de l'individu aux changements mais aussi des possibilités d'actions qu'elle offre sur cet environnement. Bien que l'on s'accorde sur l'importance de cette propriété dans l'expression d'un comportement adapté aux contraintes des situations rencontrées, il est difficile de trouver dans la littérature une définition consensuelle de cette notion. Selon les approches théoriques, on parlera de la flexibilité comme un des processus exécutifs (Rabbit, 1997; Miyake, Friedman, Emerson, Witzki et Howerter, 2000; Collette, 2004), une conduite sous dépendance des processus attentionnels (Camus, 1996) ou une composante de la pensée créative (Guilford, 1950 ; Carlier, 1973 ; Sternberg et Lubart, 1995 ; Lautrey et Lubart, 1998 ; Lubart et Lautrey, 1999 cités par Perret, 2003; Mouchiroud et Lubart, 2001; Georgsdottir et Lubart, 2003). Il ressort de l'examen de la littérature que différents facteurs contribuent à l'expression flexible des comportements et que cette notion aux acceptions multiples est surdéterminée par les tests utilisés.

L'objectif de cet article est de montrer l'intérêt des situations de résolution de problème pour opérer les distinctions nécessaires quand on parle de flexibilité. En effet, l'idée défendue est que la résolution de problème est particulièrement bien adapté pour opérationnaliser et observer les deux formes de flexibilité rapportées dans la littérature: la flexibilité spontanée et la flexibilité réactive. On montrera que l'analyse des comportements dans ces situations permet d'une part, de rendre compte des observations cliniques rapportées en neuropsychologie et des phénomènes de fixation décrits dans les travaux plus anciens des gestaltistes et d'autre part, de proposer une conception unifiée du concept de flexibilité. À cette fin, le plan de l'article est le suivant. Dans la première partie, on présente les liens entre flexibilité, rigidité et persévération. C'est notamment sous l'éclairage des concepts anciens d' «Einstellung », de rigidité et des travaux plus récents menés en neuropsychologie que l'on situe la flexibilité. Dans une deuxième partie, on présente une approche de la flexibilité dans le paradigme de la résolution de problèmes et on montre qu'il est possible d'appréhender la flexibilité et ses composantes dans les fameux problèmes de jarres de Luchins (Luchins et Luchins, 1959). Pour conclure, on discute comment le cadre conceptuel de la résolution de 
problème permet de réinterpréter les difficultés rencontrées par les sujets aussi bien dans les problèmes d'insight des gestaltistes que dans les tests de flexibilité utilisés en neuropsychologie.

\section{FLEXIBILITÉ, RIGIDITÉ ET PERSÉVÉRATION}

Qu'elle soit envisagée comme la «capacité à appliquer de manière contrôlée, différents modes de traitement à une même situation, afin de s'orienter vers le plus efficace compte tenu des caractéristiques de la tâche » (Perret, 1999), ou bien comme un des processus exécutifs « dont la fonction principale est de faciliter l'adaptation du sujet à des situations nouvelles et, ce notamment lorsque les routines d'actions, c'est-à-dire les routines sur apprises, ne peuvent suffire " (Seron, Van der Linden et, Andrès, 1999, p. 48), la flexibilité est définie comme un changement adaptatif aux évènements de l'environnement.

Par ailleurs, elle se distingue de la vicariance des différentialistes qui suppose la possession par l'individu (mais aussi par l'Espèce, voir Ohlmann, 1995) d'un répertoire de processus qui peuvent se substituer les uns les autres, des différences inter-individuelles d'évocabilité de ces processus et, selon les contextes, une variabilité intra-individuelle (Reuchlin, 1978 ; Lautrey, 1995 ; Ohlman, 1995). La flexibilité est envisagée comme la caractéristique adaptative du changement dans des situations nouvelles où les stratégies et procédures disponibles ne sont pas suffisantes pour une réponse adaptée. En ce sens, la flexibilité fait référence à la façon dont l'individu conçoit de nouvelles façons d'appréhender la situation en élaborant des procédures nouvelles qui ne font pas partie du répertoire.

\section{L'Einstellung et la rigidité}

L'étude de la rigidité des comportements a suscité un vif intérêt chez les psychologues dès les premières décennies du Xx ${ }^{\mathrm{e}}$ siècle. Caractérisant certaines pathologies, la rigidité de la conduite est néanmoins observable chez des individus sains. Ainsi, Goldstein (1943) écrivait: " Rigidity is a phenomenon which we meet again and again in pathology, but which also plays a great role in normal behavior» (p. 209). La nature et les déterminants des conduites rigides observées dans les comportements d'individus sans pathologie avérée, ont été systématiquement étudiés par Luchins (1942) lors de la résolution des fameux problèmes de jarres 
(The water-jug volume - measuring problems). Le projet initial de Luchins était d'identifier les facteurs qui augmentaient ou diminuaient les phénomènes d'habituation quand une même méthode est utilisée dans une série de problèmes similaires. Plus tard, alors qu'il est psychologue clinicien dans l'armée pendant la seconde guerre mondiale, Luchins entreprend d'utiliser ces problèmes comme des tests de la rigidité du comportement et publie, en 1950, un manuel intitulé «An examination for Flexibility-Rigidity». L'expérience de base, publiée dans la thèse de Luchins (1939), consiste à manipuler hypothétiquement des jarres de contenance différente afin d'obtenir une quantité définie. La description détaillée de l'expérience princeps sera présentée plus loin dans le texte. Toutefois, il convient ici de rappeler le principe qui sous-tend la structure des problèmes de jarres. Dans une série de problèmes similaires présentés les uns à la suite des autres, une première méthode est efficace pour résoudre les premiers problèmes et ne l'est plus pour les suivants. La thèse défendue par l'auteur est que la rigidité observée dans la résolution des problèmes - consistant à appliquer la méthode apprise alors même qu'elle s'avère inefficace - procède des effets négatifs de l'apprentissage au cours de la résolution des premiers problèmes. Les comportements de résolution de sujets sains (adultes, enfants) et pathologiques (adultes psychotiques et névrotiques) rapportés par Luchins et Luchins (1959) sont interprétés par les auteurs comme l'illustration des effets délétères de l'expérience passée, de l'habitude, et l'expression de la rigidité. Cette rigidité du comportement est définie comme la conséquence des effets de l'Einstellung, terme introduit par Warren (1934), qui désigne une certaine attitude prédisposant immédiatement l'individu à un type d'acte conscient ou moteur. Ainsi pour Luchins, les conséquences de l'habitude et des apprentissages antérieurs font partie des déterminants des comportements rigides et sont un frein à la pensée créative, pensée qui permet la découverte de solution par insight.

Cette rigidité de la conduite, ou défaut de flexibilité, a largement été décrite depuis dans les travaux menés auprès de patients cérébro-lésés, plus particulièrement chez les patients frontaux mais aussi chez des patients présentant des lésions cérébrales plus diffuses (Luria, 1980; Eslinger et Grattan, 1993; Van der Linden, Seron, Le Gall et Andrès, 1999).

\section{Les études en neuropsychologie}

Les défauts de flexibilité sont présentés par de nombreux patients qui répètent indéfiniment la même action ou le même mot et témoignent, 
comme le souligne Berthoz, (2003), d'une difficulté à se désengager de l'action en cours et à en engager une nouvelle. Cette difficulté est définie comme une conduite de persévération. "La persévération pourrait être due au maintien en mémoire du mot précédemment prononcé et qui ne serait pas assez bloqué, son seuil d'évocation restant trop bas ", (ibid., p. 90). La persévération est envisagée par l'auteur comme l'effet d'un taux d'activation trop haut de l'activité en cours. Ce taux d'activation élevé empêcherait l'expression d'une autre activité adaptée pour la situation. Cette conception est intéressante car il n'est pas nécessaire de faire appel à des défauts d'inhibition pour expliquer les conduites de persévération. Établis en fonction de la complexité du comportement affecté, l'auteur rapporte les trois types de persévération suivants : "Au niveau le plus bas, une persévération continue consiste en l'itération irrépressible de dispositifs moteurs élémentaires comme ceux que Luria avait déjà décrits en 1965, par exemple dessiner des cercles en série. Luria (1965) confirma le lien entre la persévération et les lésions du lobe frontal. À un niveau intermédiaire, une persévération récurrente consiste en la répétition de réponses préalablement produites, lorsque le patient est soumis à une séquence de stimuli par exemple. Au niveau le plus élevé, une persévération d'engluement, traduction de l'anglais stuck in set, c'est-à-dire l'incapacité de passer (switch) d'une tâche à l'autre» (ibid., pp. 90-91). L'auteur conclut que la persévération serait due à un déficit de la sélection, du choix et de l'arbitrage entre diverses actions.

Par ailleurs, des études menées auprès de patients présentant des sites lésionnels différents montrent que selon la lésion les conduites de persévération sont plus ou moins marquées. L'étude menée par Eslinger et Grattan (1993) illustre ces différences. Les auteurs ont ainsi proposé deux tests à différents groupes de patients : des patients présentant une lésion frontale, des patients atteints de lésion des ganglions de la base (infarctus ischémique), des patients présentant une lésion postérieure corticale et, des sujets sains. Ces tests sont les suivants : (1) le test du Wisconsin où il s'agit de découvrir les critères de classement de cartes et d'en changer à un moment décidé par l'examinateur (Heaton, 1981) et (2) le Alternate Uses Test qui est un test de pensée divergente où il s'agit de proposer des usages multiples d'un même objet (Guilford, Christensen, Merrifield et Wilson, 1978).

Les résultats montrent que 1) les patients frontaux sont moins performants que les patients atteints d'une lésion des ganglions de la base dans le test d'usages multiples et 2) ces deux groupes de patients présentent les mêmes scores de flexibilité dans le test du Wisconsin. Ils font plus de persévérations que les patients atteints d'une lésion postérieure 
corticale et les sujets du groupe contrôle. Les auteurs concluent que la flexibilité cognitive n'est pas un processus unitaire mais un ensemble de composantes aussi diverses que la production d'une variété d'idées, la conception d'un ensemble de réponses alternatives ou la modification de plan pour atteindre un but en fonction des changements environnementaux. Ils distinguent ainsi la flexibilité réactive et la flexibilité spontanée. La flexibilité réactive s'exprime lorsque l'environnement change et que les contraintes de la tâche exigent un changement de réponse pour une conduite adaptée. Elle est mesurée dans le test du Wisconsin. La flexibilité spontanée se manifeste quand le sujet présente des réponses variées dans un environnement stable qui ne contraint pas nécessairement au changement. Elle est mesurée dans le Alternate Uses Test. Par ailleurs, les auteurs interprètent les différences entre les patients comme l'implication différenciée des lobes frontaux et des ganglions de la base. Les lobes frontaux et les ganglions de la base seraient impliqués dans l'expression de la flexibilité réactive, les ganglions de la base n'auraient qu'un effet indirect sur la flexibilité spontanée. Une boucle corticale - sous corticale assurerait la flexibilité réactive. Les auteurs spéculent: "we believe that earlier notions of "secondary rigidity" (Goldstein, 1943) and "obstinate progression" (Villablanca et Olmstead, 1982) may be related, though more severe behavioural forms of the cognitive dissociation shown by our basal ganglia lesion group" (p. 26). Récemment, des études en imagerie cérébrale étayent cette distinction en indiquant que la flexibilité réactive dépend des régions préfrontales, pariétales et sous-corticales, alors que la flexibilité spontanée est dépendante de la portion antérieure du gyrus frontal inférieur et du noyau thalamique dorso-médian gauche (pour une revue de question, Collette, 2004).

La persévération décrite en pathologie s'apparente à la rigidité du comportement "normal » décrite par Luchins : les patients réitèrent des conduites inadaptées dans des situations qui nécessitent un changement. La différence est que dans le cas de la persévération pathologique, la conduite est déterminée par des facteurs de nature endogène (lésions cérébrales), alors que dans le cas de la rigidité du comportement « normal», la conduite est déclenchée par des facteurs conjoncturels (le contexte) de nature exogène. Dans le comportement «sain », la nature «objective» de l'attitude (Wertheimer, 1923) se développe de façon conjoncturelle dans la situation présente. Dans le comportement pathologique, il s'agit d'une attitude d'adhérence plus subjective que l'individu importe dans la situation expérimentale. 


\section{APPROCHE DE LA FLEXIBILITÉ DANS LA PROBLÉMATIQUE ACTUELLE DE LA RÉSOLUTION DE PROBLÈME}

Par opposition à la rigidité du comportement normal ou à la persévération du comportement pathologique, la flexibilité témoigne de la capacité de l'individu à changer "d'attitude » en vue de s'adapter à l'environnement dans lequel il se trouve. C'est pourquoi les situations de résolution de problème s'avèrent particulièrement bien adaptées pour appréhender la flexibilité puisqu'elles sont le prototype des situations de changement. D'autre part, les situations de problèmes imaginées par Luchins nous semblent des situations privilégiées pour appréhender au cours d'une même tâche les deux composantes de la flexibilité mises en évidence en neuropsychologie. Nous développerons cet aspect plus loin dans le texte.

\section{Flexibilité et découverte de solution}

Une situation est un problème quand on a un objectif, un but à atteindre non accessible directement, et que les moyens pour y parvenir ne sont pas disponibles en mémoire et sont à construire (Dunker, 1945 1 ). Deux grandes orientations marquent les recherches sur la résolution de problème: l'une met l'accent sur les processus de génération de solution, l'autre sur la représentation de la situation. Dans la première, la résolution de problème est envisagée comme une activité mobilisant des connaissances procédurales relatives aux savoir-faire qui se distinguent des connaissances déclaratives relatives aux savoirs (Anderson, 1983, 1993, 1995). Cette conception, largement inspirée des travaux pionniers de Newell et Simon (1972), met l'accent sur les heuristiques de résolution et les connaissances procédurales construites en situation. Dans la deuxième approche, l'éclairage est mis sur la façon dont l'individu comprend la situation compte tenu du contexte, de ses expériences passées et de ses connaissances. La découverte de solution est envisagée comme une activité guidée par l'interprétation et le codage des propriétés de la situation (par exemple, Sander et Richard, 1997 ; Sander, 2000 ; Zamani et Richard, 2000 ; Richard, Clément et Tijus, 2002). Ces propriétés sont les objets, les actions et les contraintes qui définissent la façon dont on peut agir sur les objets. C'est dans cette approche que nous envisageons la résolution de

\footnotetext{
${ }^{1}$ "A problem arises when a living creature has a goal but does not know how this goal is to be reached..." " (Dunker, 1945, p. 1).
} 
problème et proposons de définir une situation par l'ensemble des contraintes qui la caractérise. Tant que ces contraintes ne sont pas identifiées ou mal interprétées la situation est problématique. Par exemple, dans des problèmes de déplacement d'objets, tels que des problèmes de Tour de Hanoï, les contraintes qui empêchent de poser un objet sur un plus petit que lui, ou de déplacer deux objets en même temps, rendent cette situation problématique pour des enfants de 7 ans (Piaget, 1974, Richard, 1982). La même situation de déplacement d'objets dans un contexte familier d'emboîtement de gobelets de taille différente, où seule la contrainte de ne prendre qu'un seul objet à la fois doit être satisfaite, n'est pas un problème pour des enfants du même âge (Kanellaki et Richard, 1997). On peut, de cette façon, définir un problème par l'ensemble des contraintes de la situation qui sont caractéristiques du problème et qui doivent impérativement être respectées pour atteindre le but. Ce sont des contraintes exogènes ou situationnelles que doit respecter toute solution. Ce niveau de description de la situation permet de (1) distinguer la représentation de l'individu sur la situation (c'est-à-dire l'interprétation de la nature du problème et des contraintes qui permettent d'agir sur les objets) et (2) les étapes de solution choisies pour atteindre le but (c'est-à-dire les procédures mises en œuvre).

Un autre aspect à prendre en compte dans l'activité de résolution de problème est la façon dont s'opère le choix d'une stratégie, ou d'une procédure, parmi plusieurs afin d'atteindre le but. Les termes de stratégie et procédure sont souvent confondus, mais on peut opérer la distinction faite par Nguyen-Xuan (1990) : les stratégies désignent des méthodes de recherche de solution à un problème, les procédures désignent la particularisation d'une stratégie par définition d'un ensemble d'opérateurs applicables aux états de l'espace problème. En général, une situation autorise plusieurs solutions, au moins dans des systèmes relativement ouverts qui permettent plusieurs façons d'atteindre le but (Novick et Bassok, 2005). Par exemple, pour me rendre à un rendez-vous, je peux y aller à pied, prendre les transports en commun, ou bien prendre ma voiture. Le choix d'un de ces moyens de déplacement va dépendre du contexte dans lequel je me trouve. Ai-je le temps pour parcourir le trajet à pied et envie de faire un peu d'exercice? Y-a-t-il un moyen de transport qui m'amène directement sur mon lieu de rendez-vous? N'y-a-t-il pas trop d'embouteillage pour prendre ma voiture dont j'aurai besoin après mon rendezvous? etc. Dans ce cas, plusieurs possibilités sont à ma disposition. Certaines sont plus simples à mettre en œuvre, d'autres plus économiques, d'autres plus rapides. Le coût de mise en œuvre de chacune de ces solutions diffère selon les situations spécifiques rencontrées. Le choix se 
fait en fonction d'une optimisation de traitement basée sur un certain critère (simplicité, économie cognitive, rapidité). Cet exemple de la vie quotidienne illustre les situations où plusieurs modes de réalisation sont disponibles et où la résolution consiste à choisir le plus efficace dans le contexte.

Ces deux aspects de l'activité de résolution de problème - identifier les contraintes de la situation et choisir la stratégie la plus efficace dans le contexte - permettent d'envisager les différentes expressions de la flexibilité et de faire le lien avec la distinction entre flexibilité réactive et flexibilité spontanée proposée par Eslinger et Grattan (1993).

Identifier les contraintes de la situation et les respecter procède de la capacité à abandonner une stratégie inadaptée et à en élaborer une nouvelle. Ceci implique généralement une réinterprétation de la situation. De nombreuses recherches ont mis en évidence le rôle déterminant de l'interprétation du sujet dans la découverte de la solution (Richard, 2004). La représentation mentale construite en situation guide les procédures mises en œuvre par le résolveur et peut expliquer, pour une grande part, les difficultés rencontrées. On explique ainsi la difficulté des sujets comme la conséquence d'interprétations erronées de la situation qui entraînent la mise en œuvre de procédures familières, mais non adéquates, pour trouver la solution. On définit alors une situation comme une situation de résolution de problème quand le sujet est contraint de " recoder» la situation afin de trouver la solution (Richard et Zamani, 2003). Résoudre un problème c'est construire une nouvelle représentation qui sera le fruit du codage de propriétés non traitées jusque-là (Clément, 1996, 2001 ; Clément et Richard, 1997 ; Zamani, 1999, Zamani et Richard, 2000 ; Clément et Delabarre, 2001). Dans ce cas, la flexibilité correspond à un désengagement de l'action en cours pour initier une nouvelle action. Ce désengagement permet un changement de représentation sur le but qui en général se traduit par un changement de but (Richard, 1999). Dans le cas où résoudre un problème consiste à sélectionner la stratégie optimale dans le contexte, la flexibilité correspond à la capacité d'envisager plusieurs points de vue sur un même objet, c'est-àdire d'envisager plusieurs moyens pour atteindre un même but.

La distinction que nous faisons entre ces deux aspects de la résolution de problème correspond à la distinction entre flexibilité réactive et flexibilité spontanée proposée par Eslinger et Grattan (1993). Dans la résolution de problème, les stratégies mises en œuvre au début de la résolution sont très souvent des stratégies qui se sont avérés efficaces dans des problèmes similaires et qui sont appliquées par transfert analogique. Les processus sont de nature automatique et l'attention est guidée par les données. Dans 
certains cas, ces stratégies peuvent s'avérer inefficaces et conduire en impasse. C'est dans l'impasse, quand plus aucune action ne semble possible, que la flexibilité réactive peut se manifester. Elle procède d'une réorientation de l'attention déclenchée par un signal de l'environnement vers des propriétés qui n'étaient pas dans le focus attentionnel. Des processus sous contrôle volontaire permettent alors le changement de but et la sortie d'impasse. La flexibilité spontanée se manifestant par un changement non contraint par la situation correspond au choix d'une stratégie optimale pour la tâche. Ce choix non dépendant d'un signal de l'environnement est possible parce qu'on s'est désengagé de l'action. Dans ce cas, l'activité est orientée par des processus contrôlés. Le codage des caractéristiques pertinentes de la situation permet d'envisager différents moyens pour atteindre le but et le choix de la stratégie la plus efficace et adaptée à la situation (c'est-à-dire, selon le contexte, la plus rapide, la plus économique cognitivement ou la plus simple à mettre en cuvre). Cette composante de la flexibilité témoigne de la capacité d'anticiper hors action plusieurs points de vue sur un même objet. Cette anticipation facilite le passage d'une stratégie à l'autre. Elle relève d'une compréhension conceptuelle de la tâche et permet d'éliminer les stratégies les moins efficaces et de découvrir les plus efficientes (Siegler, 1999).

\section{Mise en évidence des différentes composantes de la flexibilité dans la résolution des problèmes de jarres}

Les problèmes de jarres imaginés par Luchins sont un bon exemple de problèmes pour identifier l'expression de la flexibilité spontanée, de la flexibilité réactive et des persévérations. Avant de présenter l'analyse que nous proposons de ces problèmes, revenons sur l'expérience princeps et les résultats rapportés par Luchins et Luchins (1959). Les consignes ${ }^{1}$ et les problèmes sont les suivants :

"Il s'agit de résoudre des problèmes dans lesquels il faut obtenir une certaine quantité en manipulant (hypothétiquement) des jarres de capacités définies. Imaginez-vous près d'une source d'eau comme le robinet d'une cuisine ou près d'un puits. On vous donne une jarre vide qui a une contenance de 29 quarts et une autre de capacité de 3 quarts; vous devez obtenir 20 quarts d'eau. Souvenez-vous que les jarres ne sont pas graduées. La seule chose que vous sachiez est que si vous remplissez jusqu'au

${ }^{1}$ Notre traduction d'après Luchins et Luchins 1959, p. 108-109. 
bord la première vous obtenez 29 quarts et la seconde 3 quarts. Commencez par remplir la jarre de 29 quarts; puis utilisez la jarre vide de 3 quarts trois fois de suite pour enlever les 9 quarts excédants. Chaque fois videz le contenu de la petite jarre dans l'évier ou dans le puits.

Maintenant essayez ce problème : étant données une jarre de 21 quarts, une autre de 127 quarts, et une troisième de 3 quarts, vous devez obtenir 100 quarts. Donnez-vous un maximum de deux minutes et demi pour ce problème puis continuez à lire.

Une façon de résoudre ce problème est la suivante: D'abord, remplissez la jarre de 127 quarts, ce qui fera un excédant de 27 quarts. De cette jarre, remplissez la jarre de 21 quarts. L'excès d'eau restante dans la jarre de 127 n'est plus que de 6 quarts. Pour enlever le trop plein, remplissez deux fois la jarre de 3 quarts. La jarre la plus grande contient maintenant 100 quarts. On peut exprimer la solution de la façon suivante : $127-21-$ $3-3=100$, ou $127-3-3-21=100$ ' ou $127-3-21-3=100$.

Le problème peut aussi se résoudre en utilisant seulement la jarre de 127 quarts et celle de 3 quarts. Remplissez la jarre de 127 quarts et enlevez le trop plein de 27 quarts en retirant neuf fois la jarre de 3 quarts; il reste 100 quarts dans la grande jarre ; $127-9 \times 3=100$.

Maintenant essayez de résoudre les onze problèmes suivants [Tableau I]. Pour obtenir le volume désiré, utilisez certaines ou toutes les jarres disponibles. Vous verrez que les deux premiers problèmes sont ceux que l'on vient de présenter. Si vous ne pouvez pas résoudre un problème dans les deux minutes et demi passez au problème suivant. Vous pouvez écrire verbalement votre solution, ou utiliser des symboles mathématiques dans une équation, ou dessiner des traits pour figurer le remplissage et le vidage des jarres ».

Si on analyse les problèmes du point de vue de la procédure de résolution, leur particularité est que les problèmes 2,3 , 4, et 6 se résolvent tous par une même méthode : $\mathrm{B}-\mathrm{A}-2 \mathrm{C}$ (Les jarres sont respectivement désignées de gauche à droite par A, B, C). Cette solution et des solutions plus simples peuvent être envisagées pour les problèmes suivants : pour le problème 5 , la solution $\mathrm{A}+\mathrm{C}+\mathrm{C}$; pour les problèmes 7 et 11 , la solution $\mathrm{A}-\mathrm{C}$; pour les problèmes 8 et 10 , la solution $A+C$. Seul le problème 9 se résout d'une seule façon, la solution simple A - C.

De l'expérience originale (Luchins, 1939), Luchins et Luchins (1959) rapportent que sur les 1039 sujets qui ont participé à l'expérience, $83 \%$ utilisent la solution $\mathrm{B}-\mathrm{A}-2 \mathrm{C}$ dans les problèmes 7 et 8 et $64 \%$ d'entre eux échouent au problème 9 . En revanche, dans le groupe contrôle des 970 sujets qui ont résolu directement les problèmes 7 à 11 , moins de $1 \%$ utilise la méthode $\mathrm{B}-\mathrm{A}-2 \mathrm{C}$ et seulement $5 \%$ des sujets échouent au 
Tableau I. L'expérience princeps des problèmes de jarres d'après Luchins et Luchins, 1959.

Table I. The water-jug volume-measuring problems (Luchins et Luchins, 1959)

\begin{tabular}{ccrcr}
\hline Problème & $\begin{array}{c}\text { Les jarres } \\
\text { et leurs } \\
\text { contenances }\end{array}$ & & $\begin{array}{c}\text { Solution } \\
\text { à obtenir }\end{array}$ \\
\hline 1 & $\cdots$ & 29 & 3 & 20 \\
2 & 21 & 127 & 3 & 100 \\
3 & 14 & 163 & 25 & 99 \\
4 & 18 & 43 & 10 & 5 \\
5 & 9 & 42 & 6 & 21 \\
6 & 20 & 59 & 4 & 31 \\
7 & 23 & 49 & 3 & 20 \\
8 & 15 & 39 & 3 & 18 \\
9 & 28 & 76 & 3 & 25 \\
10 & 18 & 48 & 4 & 22 \\
11 & 14 & 36 & 8 & 6 \\
\hline
\end{tabular}

problème 9. Les auteurs commentent : "La majorité des sujets adhère à un mode de solution et ne change pas pour une solution plus simple, même quand la méthode à laquelle ils sont habitués n'est pas adéquate » ${ }^{1}$ (p. 110). Ils interprètent ces résultats comme le fait d'une mécanisation de la pensée.

Si on analyse les problèmes non seulement du point de vue de la procédure (les combinaisons de jarres à effectuer) mais aussi du point de vue des propriétés des problèmes (le nombre de jarres à utiliser), on note qu'au changement de procédure correspond un changement du nombre de jarres à utiliser. En effet, de ce point de vue on peut remarquer que la contrainte de situation des problèmes 2, 3, 4 et 6 est qu'il faut utiliser les trois jarres A, B et C. Dans les problèmes 5, 7, 8, 10 et 11 , cette contrainte reste pertinente mais une autre contrainte l'est aussi : on peut utiliser les deux jarres A et C. Dans le problème 9 , seule cette dernière contrainte est

${ }^{1}$ The majority of the subjects rigidly adhered to one mode of solution and did not shift to a simpler method even when the method to which they had become habituated was no longer adequate" (Luchins et Luchins, $1959, \mathrm{p} 110)$ 
pertinente: il faut utiliser les deux jarres A et C. Ici, le changement de codage correspond au traitement de la propriété pertinente des problèmes qui est le nombre de jarres impliqué dans la solution. L'effet d'attitude et le transfert de procédure décrits par Luchins peuvent s'expliquer par la façon dont on code la situation.

La flexibilité liée à la sélection d'une procédure la plus adaptée peut s'exprimer dès les problèmes 5,7 ou 8 par la découverte d'une solution à deux jarres. La flexibilité liée à la contrainte situationnelle s'exprimera dans le problème 9 . En effet, si on se trouve en impasse au problème 9 , on peut faire le constat que la procédure qu'on a utilisée au cours des sept problèmes précédents est inadaptée et rechercher une autre procédure. En revanche, découvrir la procédure plus simple à deux jarres dès les problèmes 5,7 ou 8 implique que l'on se soit engagé spontanément dans la recherche d'une autre procédure. Cette recherche procède d'une analyse des caractéristiques de chacun des problèmes puisque rien dans la situation ne contraint à abandonner une procédure efficace jusqu'à présent. Dans ce cas, la recherche d'autres solutions possibles à un problème suppose que le but soit représenté de façon autonome et non associée à la procédure.

D'autre part, en distinguant les propriétés du problème des procédures elles - mêmes, on peut différencier les recherches de solution qui procèdent d'un simple changement de procédure et celles qui correspondent à un changement de codage. En effet, sachant qu'il faut utiliser deux ou trois jarres dans ces problèmes, il y a plusieurs combinaisons à tester. Dans le problème 9 , deux stratégies de recherche de solution peuvent être envisagées : celle qui consiste en des combinaisons de deux jarres et celle qui consiste en des combinaisons de trois jarres. La première stratégie relève d'un changement de procédure consécutif à un changement de codage des propriétés du problème. La seconde correspond à un changement de procédure sans changement de codage sur les propriétés.

Dans une recherche récente sur l'involution de la flexibilité cognitive, on a pu observer les deux profils de recherche de solution aux problèmes 7 et 8: une recherche de solution à deux jarres ou bien l'application de la solution à trois jarres dans ces deux problèmes. Au problème 7 , les sujets qui recherchent une solution optimale découvrent que le nombre de jarres à utiliser n'est pas le même sur l'ensemble des problèmes. Ils ont changé de représentation avant d'être confrontés à l'impasse en s'engageant dans le codage des propriétés des problèmes. D'autre part, l'analyse des recherches de solution à trois jarres dans le problème 9 a permis de dégager deux nouveaux profils en situation d'impasse (échec de la solution à trois jarres). Pour certains sujets, le constat d'inefficacité de la 
solution à trois jarres permet l'orientation de l'attention sur une caractéristique des problèmes qu'ils n'avaient pas traitée jusque-là : après une première tentative infructueuse, ils découvrent la solution à deux jarres. Les autres essaient de nouvelles solutions à trois jarres sans découvrir la solution à deux jarres. Pour ces sujets, l'impasse est une occasion de changer de procédure mais pas de changer de codage sur la situation : ils essaient de nouvelles combinaisons à trois jarres. Ce n'est qu'en donnant une information explicite sur le changement à opérer et sur les propriétés pertinentes à prendre en compte, que ces sujets découvrent la solution à deux jarres. Enfin, certains sujets présentent des conduites de persévération au cours du problème 9 . Les informations explicites ne les aident pas à trouver la solution : ils continuent à utiliser la combinaison précédente à trois jarres (Guédin et Clément, 2005).

Cette situation expérimentale est très riche car elle permet d'observer différents niveaux de flexibilité.

Le premier correspond à la flexibilité qui ne dépend d'aucun signal direct provenant de la situation. Il suppose une capacité à adopter différents points de vue sur un même objet en choisissant le plus efficient (simplicité de la procédure). Cette flexibilité est mise en œuvre de façon relativement explicite: les sujets s'engagent dès les premiers problèmes dans le codage de la situation en testant plusieurs combinaisons de jarres. Le second niveau correspond à la flexibilité déclenchée par un signal de la situation: l'impasse. Il peut être mis en œuvre de façon relativement implicite. On peut en distinguer deux formes : la plus élaborée est la capacité à remettre en question l'interprétation de la situation et à procéder à une nouvelle analyse en recodant les propriétés de la situation. Dans ce cas, le constat d'impasse est suffisant pour changer de représentation et de stratégie. L'autre forme est la capacité à découvrir une autre procédure sans s'engager dans un recodage de la situation. Ce n'est qu'en orientant l'attention des sujets sur les propriétés pertinentes qu'on les aide à découvrir la solution.

\section{DISCUSSION ET CONCLUSION}

L'intérêt suscité par les aspects flexibles et rigides de nos comportements n'a pas faibli au cours du temps. Il a entraîné très tôt des débats et des points de vue différents sur la nature de la rigidité de la conduite (Lewin, 1935, 1936; Werner, 1940 ; Golstein, 1943 ; Luchins et Luchins, 1959). 
L'examen de la littérature plus récente montre que 1) le concept est surdéterminé par les épreuves dans lesquelles on mesure la flexibilité et 2) il renvoie à des processus différents selon les orientations théoriques. La nature de la flexibilité et son caractère unitaire restent une question ouverte, même si la distinction introduite par Eslinger et Grattan (1993) ouvre de nouvelles perspectives. En effet, quand on cherche à décrire des comportements flexibles, il est important d'identifier les différents niveaux de flexibilité. Nous avons montré qu'en utilisant une situation de résolution de problème appropriée, il était possible de mettre en évidence au sein d'une même tâche ces différents niveaux.

La première forme de flexibilité, ne dépendant d'aucun signal de la situation, est sous-tendue par des processus descendants dirigés par le but et sous contrôle volontaire. La deuxième forme, déclenchée en impasse, est guidée par des processus ascendants. Dans ce cas, les propriétés de la situation capturent l'attention et permettent de changer de représentation. En impasse, deux changements sont possibles: soit on change de procédure sans s'engager dans le recodage des propriétés de la situation et la stratégie mise en ouvre n'est pas adaptée. Soit on s'engage dans le recodage qui permet de changer de stratégie et de trouver une procédure adaptée. Ces deux formes de flexibilité, décrites ici comme une flexibilité indépendante d'une alerte provenant de la situation et une flexibilité déclenchée par un signal de l'environnement, sont sans doute liées aux deux formes d'orientation de l'attention, endogène et exogène, décrites dans le cadre général des mécanismes attentionnels (voir pour une synthèse des approches théoriques de l'attention, Siéroff, 1998; Camus 2003).

D'autre part, l'approche de la flexibilité que nous proposons permet de réinterpréter les phénomènes de fixation décrits par les gestaltistes et les comportements de persévération rapportés en neuropsychologie.

Les gestaltistes ont décrit la flexibilité comme un phénomène lié à l'insight et antagoniste des phénomènes de fixation. La fixation peut être liée à trois causes : la mécanisation de la pensée, la prégnance de la bonne forme et la fixité fonctionnelle (voir pour une synthèse des travaux de la Gestalt, Murray, 1995). La première, largement décrite dans cette revue de question, est l'effet de l'« Einstellung ». La seconde est liée à la difficulté à déstructurer perceptivement la bonne forme de la stimulation. Le problème des neuf points illustre ce frein à la découverte de la solution. (Dominowski, 1981; Weisberg et Alba, 1981, a, b ; 1982 ; Ellen, 1982). La troisième source de fixation est la difficulté à utiliser un objet dans une fonction non habituelle (par exemple, le problème des cordes de Maier, 1945). On peut réinterpréter la découverte de solution dans ces problèmes 
d'insight comme la mise en jeu, dans tous les cas, de la flexibilité qui ne correspond pas seulement à un changement de procédure mais à un changement de représentation sur le problème. Dans les problèmes de jarres, on l'a vu, c'est la résolution des premiers problèmes qui induit une interprétation erronée et conduit en impasse. Il faut abandonner cette interprétation pour trouver la solution au problème critique. Dans le problème des 9 points, c'est la conjonction des propriétés de la situation (la bonne forme) et des savoirs en mémoire qui induit une interprétation erronée : les sujets interprètent «joindre les points» comme «aller d'un point à l'autre ». Cette représentation prototypique de l'action de joindre des points interdit de dépasser les points (Richard, 1994). Les phénomènes de fixité fonctionnelle sont du même type: le changement de représentation est difficile car ce qui est saillant c'est la propriété fonctionnelle habituelle de l'objet et non une propriété que peut remplir une autre fonction.

Concernant la perspective clinique, on propose ici une assise conceptuelle à la distinction entre flexibilité réactive et flexibilité spontanée. De plus, ce cadre conceptuel permet la mise en lien entre les problèmes d'insight des gestaltistes et les tests neuropsychologiques de la flexibilité.

Nous proposons de réinterpréter les épreuves utilisées en neuropsychologie du point de vue de la nature de la flexibilité et du changement opéré. Dans le test du Wisconsin, il s'agit de la flexibilité réactive et on peut penser à un changement de représentation plutôt qu'à l'application d'une nouvelle procédure. En effet, dans cette tâche le changement d'indice est relativement explicite. Les tests de pensée divergente concernant des utilisations inhabituelles d'objets sont à relier à la fixité fonctionnelle des gestaltistes. Toutefois, ces tests sont des épreuves explicites de productions d'idées et non des tâches implicites d'apprentissage par l'action dans lesquelles c'est l'action et l'évaluation de ses résultats qui permettent le changement de représentation (Gick et McGarry, 1992; Reber et Kotovsky, 1997). Ces tests de pensée divergente ont à affaire avec la flexibilité spontanée et il s'agit d'un changement de représentation sur les informations fournies dans la situation. Enfin, dans les tests de flexibilité de clôture (Witkin, Oltman et Karp, 1971) c'est la structure perceptive qui rend difficile le changement de point de vue. Ce changement est lié à la flexibilité spontanée car la découverte d'une forme simple dans une forme complexe procède d'une recherche délibérée et explicite. Dans ces tests, il s'agit aussi d'un changement de représentation.

En conclusion, l'examen de la littérature sur la flexibilité montre que cette notion doit être conceptualisée dans le cadre plus général d'une approche s'attachant à appréhender l'activité de l'individu en terme de processus. 
Cette approche suppose que des distinctions entre les différentes formes flexibilité soient envisagées et que les changements qui s'opèrent soient analysés du point de vue de leur nature. Par ailleurs, ceci implique qu'une analyse de la tâche et des tests utilisés soit menée sur les processus impliqués dans leur réalisation. Cette approche permet de catégoriser les différentes épreuves et de différencier les difficultés des sujets.

\section{BIBLIOGRAPHIE}

Anderson, J.R. (1983). The architecture of cognition. Cambridge, MA: Harvard University Press.

Anderson, J.R. (1993). Rules of mind. Hillsdale, NJ: Lawrence Erlbaum.

Anderson, J.R. (1995). Cognitive psychology an its implications, fourth ed. New York: Freeman \& Company.

Berthoz, A. (2003). La décision. Paris: Odile Jacob.

Camus, J.F. (1996). La psychologie cognitive de l'attention. Paris : Armand Colin.

Camus, J.F. (2003). L'attention et ses modèles. Psychologie Française, 48(1), 5-18.

Carlier, M. (1973). Étude différentielle d'une modalité de la créativité : la flexibilité. Monographies françaises de psychologie, 25. Paris : CNRS.

Clément, E. (1996). L'effet du contexte sémantique dans l'élaboration de la représentation du problème. L'Année Psychologique, 96, 409-442.

Clément, E. (2001). Étude des différences de flexibilité mentale dans l'activité de résolution de problèmes. In A. Flieller, C. Bocéréan, J.L. Kop, E. Thiébaut, A.M. Toniolo \& J. Tournois (Eds.), Questions de psychologie différentielle. Rennes: Presse Universitaire de Rennes.

Clément, E., \& Delabarre, S. (2001). Différences intergénérationnelles dans la résolution de problèmes. In A. Flieller, C. Bocéréan, J.L. Kop, E. Thiébaut, A.M. Toniolo \&
J. Tournois (Eds.), Questions de psychologie différentielle. Rennes: Presse Universitaire de Rennes.

Clément, E., \& Richard, J.F. (1997). Knowledge of domain effects in problem representation: the case of Tower of Hanoi isomorphs. Thinking and Reasoning, 3(2), 133-157.

Collette, F. (2004). Exploration des fonctions exécutives par imagerie cérébrale. In T. Meulemans, F. Collette \& M. Van der Linden (Eds.), Neuropsychologie des fonctions exécutives. Marseille: Solal.

Dominowski, R.L. (1981). Comment on an examination of the alleged role of «fixation » in the solution of several insight problems by Weisberg and Alba. Journal of Experimental Psychology: General, 110, 193-198.

Dunker, K. (1945). On problem-solving (L. S. Lees, Trans.). Psychological Monographs, 58 (Vhole No. 270).

Ellen, P. (1982). Direction, past experience and hints in creative problem solving: reply to Weisberg and Alba. Journal of Experimental Psychology: General, 111, 316-325. Eslinger, P.J., \& Grattan, L.M. (1993). Frontal lobe and frontal - striatal substrates for different forms of human cognitive flexibility. Neuropsychologia, 31, 17-28.

Georgsdottir, A.S., \& Lubart, T.I. (2003). La flexibilité cognitive et la créativité : une approche développementale, différentielle 
et expérimentale. Psychologie Française, 48(3), 29-40.

Gick, M.L., \& Mc Garry S.J. (1992). Learning from mistakes: inducing analogous solution failures to a source problem produces later successes in analogical transfer. Journal of Experimental Psychology: Learning, Memory, and Cognition, 18, 623-639.

Goldstein, K. (1943). Concerning rigidity. Character and Personality, 11, 209-226.

Guédin, C., \& Clément, E. (2005). Devenir de la flexibilité au cours du vieillissement normal. In B. Vivicorsi \& R. Collet (Eds.), Handicap, cognition et prise en charge individuelle: des aspects de la recherche au respect de la personne. Rouen : Presses Universitaires de Rouen et du Havre.

Guilford, J.P. (1950). Creativity. American Psychologist, 5, 444 - 454.

Guilford, J.P., Christensen, P.R., Merrifield, P.R., \& Wilson, R.C. (1978). Alternate Uses: Manual of instructions and Interpretations. Sheridan Psychological Services: Orange, CA.

Heaton, R. K. (1981). Wisconsin Card Sorting Test Manual. Psychological Assessment Resources, Inc.: Odessa, FL.

Kanellaki-Agathos, S., \& Richard, J. F. (1997). Planification et représentation de l'action chez l'enfant. Archives de Psychologie, 65, 49-76.

Lautrey, J. (1995). Universel et Différentiel en psychologie. Paris : PUF.

Lautrey, J., \& Lubart, T.I. (1998). Créativité. In O. Houdé, D. Kayser, O. Koening, J. Prouste \& F. Rastier (Eds.), Vocabulaire de Sciences Cognitives. Paris : PUF.

Lewin, K. (1935). A dynamic theory of personality. New York: McGraw-Hill.

Lewin, K. (1936). Principles of topological psychology. New York: McGraw-Hill.

Lubart, T., \& Lautrey, J. (1999). Créativité, flexibilité de pensée et rôle des processus d'inhibition. Communication orale au colloque «Rationalités en développement». Aix-en Provence: France.
Luchins, A.S. (1939). The Einstellung effect in learning by repetition. Unpublished doctoral dissertation, New York University.

Luchins, A.S. (1942). Mechanization in problem solving. Psychological Monograph, $54, \mathrm{n}^{\circ} 6$ (Whole $\mathrm{N}^{\circ} 248$ ).

Luchins, A.S. (1950). An examination for flexibility-rigidity. Montrose, N.Y.: Veterans Admin.

Luchins, A.S., \& Luchins, E.H. (1959). Rigidity of behavior. A variational approach to the effect of Einstellung. University of Oregon Monographs, Studies in Psychology $\mathrm{N}^{\circ} 3$.

Luria, A.R. (1965). Two kinds of motor perseveration in massive injury of the frontal lobe. Brain, 88, 110.

Luria, A.R. (1980). Higher cortical functions in man, Second edition, revised and expanded. New York: Basic Books, Inc, Publishers.

Maier, N.R.F. (1945). Reasoning in humans: III. The mechanics of equivalent stimuli and of reasoning. Journal of Experimental Psychology, 35, 349-360.

Miyake, A., Friedman, N.P., Emerson, M.J., Witzki, A.H., \& Howerter, A. (2000). The unity and diversity of executive functions and their contribution to complex "frontal lobe" tasks: a latent variable analysis. Cognitive Psychology, 41, 49-100.

Mouchiroud, C., \& Lubart, T.I. (2001). Children's original thinking: An empirical examination of alternative measures derived from divergent thinking tasks. Journal of Genetic Psychology, 162 (4), 382-401.

Murray, D.J. (1995). Gestalt psychology and the cognitive revolution. London: Harvester Wheatsheaf.

Newell, A., \& Simon, H.A. (1972). Human Problem Solving. Englewood Cliffs (NJ): Prentice Hall.

Nguyen-Xuan, A. (1990). Conscience, prise de conscience et métacognition. In J.F. Richard, C. Bonnet \& R. Ghiglione (Eds.), Traité de psychologie cognitive. Paris : Dunod. 
Novick, R.L., \& Bassok, M. (2005). Problem solving. In K.J. Holyoak \& R.G. Morrison (Eds.), The Cambridge Handbook of Thinking and reasoning. New York: Cambridge University Press.

Ohlmann, T. (1995). Processus vicariants et théorie neutraliste de l'évolution: une nécessaire convergence. In J. Lautrey (Ed.), Universel et différentiel en psychologie. Paris : PUF.

Perret, P. (1999). La flexibilité cognitive: une propriété émergente du fonctionnement. rapport polycopié, Université d'Aix-en Provence.

Perret, P. (2003). Contrôle inhibiteur et développement cognitif : perspectives actuelles. Revue de Neuropsychologie, 13, 345-373.

Piaget, J. (1974). La prise de conscience. Paris : PUF.

Rabbit, P. (1997). Methodology of frontal and executive function. Hove: Psychology Press Publishers.

Reber, P.J., \& Kotovsky, K. (1997). Implicit learning in problem solving: The role of working memory capacity. Journal of Experimental Psychology: General, 126(2), 178-203.

Reuchlin, M. (1978). Processus vicariants et différences interindividuelles. Journal de Psychologie, 2, 133-145.

Richard, J.F. (1982). Planification et organisation des actions dans la résolution du problème de la Tour de Hanoï par des enfants de 7 ans. L'Année Psychologique, 82, 307-336.

Richard, J.F. (1994). La résolution de problèmes. In M. Richelle, J. Requin \& M. Robert (Eds.), Traité de psychologie Expérimentale. Paris : PUF.

Richard, J.F. (1999). Comportements, buts et représentations. Psychologie Française, 44, 75-90.

Richard, J.F. (2004). Les activités mentales. Paris : Armand Colin.

Richard, J.F., Clément, E, \& Tijus, C. (2002). Les composantes sémantiques dans la résolution de problèmes isomorphes. Revue d'Intelligence Artificielle, 16, 191-219.
Richard, J.F., \& Zamani, M. (2003). A Problem-Solving Model as a Tool for Analyzing Adaptive Behavior. In R.J. Sternberg, J. Lautrey, \& T. Lubart (Eds.), Models of Intelligence: An international perspective. Washington, DC: American Psychological Association.

Sander, E. (2000). L'analogie, du naïf au créatif. Paris : L'Harmattan.

Sander, E., \& Richard J.F. (1997). Analogical transfer as guided by an abstraction process: The case of learning by doing text editing. Journal of Experimental Psychology: Learning, Memory and Cognition, 23, 1459-1483.

Seron, X., Van der Linden, M., \& Andrès, P. (1999). Le lobe frontal: à la recherche de ses spécificités fonctionnelles. In M. Van der Linden, X. Seron, D. Le Gall \& P. Andrés (Eds.), Neuropsychologie des lobes frontaux. Marseille : Solal.

Siegler, R.S. (1999). Strategic development. Trends in Cognitive Sciences, 3, 430-435.

Siéroff, E. (1998). Théories et paradigmes expérimentaux de l'attention. Revue de Neuropsychologie, 8, 3-14.

Sternberg, R.J., \& Lubart, T.I. (1995). Defining the crowd. New York: Free Press.

Van der Linden, M., Seron, X., Le Gall, D., \& Andrès, P. (1999). Neuropsychologie des lobes frontaux. Marseille : Solal.

Villablanca, J.R., \& Olmstead, C.E. (1982). The striatum: A fine turner of the brain. Acta Neurobiol. Exp. 42, 227- 299.

Warren, H.C. (1934). Dictionnary of Psychology. New York: Houghton Mifflin.

Werner, H. (1940). The concept of rigidity: A critical evaluation. Psychological Review, 53, 43-52.

Weisberg, R.W., \& Alba, J.W. (1981a). An examination of the alleged role of «fixation » in the solution of several «insight » problems. Journal of Experimental Psychology: General, 110, 169-192.

Weisberg, R.W., \& Alba J.W. (1981b). Gestalt theory, insight and past experience: 
reply to Dominovski. Journal of Experimental Psychology: General, 110, 193-198.

Weisberg, R.W., \& Alba, J.W. (1982). Problem solving is not like perception: More on Gestalt theory. Journal of Experimental Psychology: General, 111, 326-330.

Wertheimer, M. (1923). Untersuchungen zur Lehr von der Gestalt, II. Psychol. Forsch., 4: 301- 350. Condensed in Section 5. In W. D. Ellis (Ed.), 1938, A source book of Gestalt psychology. New York: Harcourt, Brace.
Witkin, H.A., Oltman, P.K., \& Karp, S.A. (1971). Manuel for embedded figures test, children's embedded figures test, and group embedded figures test. Palo-Alto, CA: Consulting Psychologists Press.

Zamani, M. (1999). La découverte des propriétés pertinentes de la tâche: exemples du test $d u$ passalong. Thèse d'université de Paris 8 .

Zamani, M., \& Richard, J.F. (2000) Object encoding, goal similarity, and analogical transfer. Memory and Cognition, 28(5), 873-886. 